\title{
Identification of barriers for the adherence to the nutritional plan in patients with type 2 diabetes
}

\author{
García-Ulloa AC, Landa-Anell MV, Velázquez-Jurado HR, Melgarejo-Hernández M, \\ Ovalle-Escalera CN, Ramírez-García JA, Hernández-Jiménez SC. \\ Centro de Atención Integral del Paciente con Diabetes (CAIPaDi) \\ Instituto Nacional de Ciencias Médicas y Nutrición Salvador Zubirán. Mexico City.
}

Background: The assessment and diminishment of patients' barriers is important to promote their adherence to the nutritional plan.

Aims: To identify the most common barriers for nutritional adherence in patients with type 2 diabetes receiving multidisciplinary care in our program.

Methods: The CAIPaDi program addresses patients with type 2 diabetes with less than 5 years of diagnosis, non-smokers, and without incapacitating chronic complications. The intervention consists of four monthly visits. In every visit, patients are evaluated by nurses, endocrinologists, nutritionists, diabetes educators, psychologist, psychiatrist, foot care, dentists, ophthalmologist and physical therapists. The nutritional intervention is structured to carry out nutrition educational strategies in each visit through a specific topic and printed material to solve each of the different barriers. We applied an instrument to evaluate Barriers for the Nutritional Follow-Up (BSN). This tool was designed between five experts (three nutritionists, an endocrinologist and a psychologist). The barriers included were the most commonly reported by patients and others mentioned in the literature: lack of time, I didn't understand the instructions, economic situation, lack of time to prepare my meals, most of my meals are outside from home, denial or rejection to modify my nutritional habits and none. Figure 1 shows visual information given to patients to help them diminish the different barriers reported during the program and help adhere to the nutritional plan.
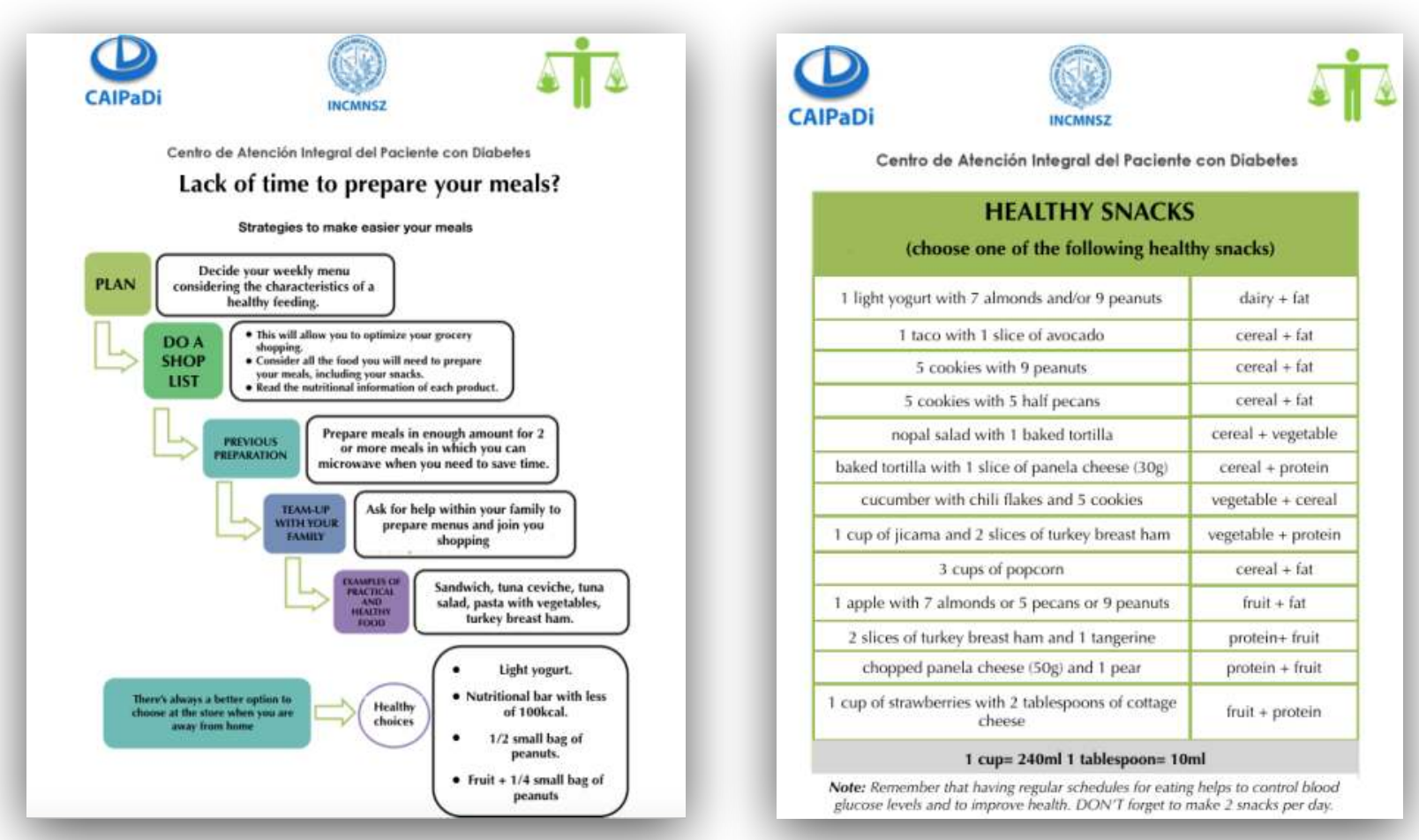

Figure 1: Visual information given to patients to diminish barriers and improve adherence to the nutritional plan

Results: We included 513 patients with an average age of 52 years and an median time of diagnosis of $3(0-5)$ years, $60.4 \%$ women. At the beginning, $78.9 \%$ of the participants stated a barrier. The most common barriers were "lack of information" (about proper eating) (28.5\%), "most of my meals are outside from home" (15.4\%) and "lack of time to prepare my meals" (14.8\%).
The proportion of each barrier according to the first and last phase of the treatment was stated. We found statistical significance from the first to the last visit $(p<0.001)$. After the structured nutritional intervention, $48.9 \%$ of the patients continued having a nutritional barrier, being "lack of information" the one with the most significant change $(p<0.001)$ (less patients reporting the barrier). The barrier "most of my meals are outside from home" was reported by more patients in the fourth visit. Table 1 shows the changes in barriers after the nutritional intervention. Table 2 shows the metabolic changes at the beginning and at the end of the 4th month, after receiving a multidisciplinary intervention.

\begin{tabular}{|l|c|c|c|}
\hline \multicolumn{1}{|c|}{ Barrier } & V1 (\%) & V4 (\%) & p \\
\hline Lack of information & 28.5 & 1.8 & $<0.001$ \\
\hline Did not understand indications & 1.35 & 0 & $<0.001$ \\
\hline Economical situation & 5.9 & 2.7 & $<0.001$ \\
\hline Lack of time to prepare meals & 14.8 & 12.2 & $<0.001$ \\
\hline Eat mostly out from home & 15.4 & 19.4 & $<0.001$ \\
\hline Denial to make changes & 14.4 & 12.6 & $<0.001$ \\
\hline None & 21.1 & 51.1 & $<0.001$ \\
\hline
\end{tabular}

Table 1: Comparison of barriers for adherence to the nutritional plan before and after nutritional intervention.

\begin{tabular}{|c|c|c|c|}
\hline & $\mathbf{V 1}$ & $\mathbf{V 4}$ & $\mathbf{p}$ \\
\hline Blood glucose (mg/dl) & $157.01(105-181)$ & $99.34(88-117)$ & 0.000 \\
\hline HbA1c (\%) & $8.15 \pm 2.42$ & $6.36 \pm 0.96$ & 0.000 \\
\hline Triglycerides (mg/dl) & $224.94(124-253)$ & $127.86(91-147)$ & 0.000 \\
\hline LDL cholesterol (mg/dl) & $113.62(89-138)$ & $86.47(67-102)$ & 0.000 \\
\hline No-HDL cholesterol (mg/dl) & $152.1(123-176)$ & $106.17(84-122)$ & 0.000 \\
\hline Barriers (number of) & $4(1-6)$ & $6(5-7)$ & 0.000 \\
\hline
\end{tabular}

Table 2: Comparison of metabolic parameters between first and fourth visit.

Discussion. Most people understand the benefits of a healthy diet, however some of them do not manage to achieve long term changes even after receiving specific therapeutic recommendations for type 2 diabetes control. This can be a result of different barriers that hinder the attainment of a healthy nutritional plan. In the present study, more than $50 \%$ of the patients end the program without any nutritional barrier, nevertheless the barrier "most of my meals are outside from home" increases from $15.4 \%$ in the first visit to $18.5 \%$ in visit 4 . A reason for this raise may be the recognition of the barrier after each nutritional intervention.

The reduction of each barrier can induce to more sustainable nutritional changes in a short and long term. Its solution through feasible strategies allows a better adherence to the nutritional advices.

\section{Reference:}

Sergio Hernández-Jiménez, Cristina García-Ulloa, Roopa Mehta, Carlos A. Aguilar-Salinas and David Kershenobich-Stalnikowitz. Innovative Models for the Empowerment of Patients with Type 2 Diabetes: The CAIPaDi Program. Recent Pat Endocr Metab Immune Drug Discov 2014; 8(3):202-9.

The CAIPaDi program has received grants from Astra Zeneca, Fundación Conde de Valenciana, Novartis, Consejo Nacional de Ciencia y Tecnología, Nutrición Médica y Tecnología, NovoNordisk, Boehringer Ingelheim, Dirección General de Calidad y Educación en Salud, Eli Lilly, Merck Serono, MSD, Silanes, Chinoin and Carlos Slim Health Institute. There are no other potential conflicts of interest relevant to this work. 